\title{
Comparison of IgA, IgG and neutralizing antibody responses following immunization with Moderna, BioNTech, AstraZeneca, Sputnik-V, Johnson and Johnson, and Sinopharm's COVID-19 vaccines.
}

\section{Tomabu Adjobimey ( $\square$ Tomabu.Adjobimey@ukb-uni-bonn.de )}

Institute of Medical Microbiology, Immunology and Parasitology (IMMIP), University Hospital Bonn, Bonn, Germany

\section{Julia Meyer}

Institute of Medical Microbiology, Immunology and Parasitology (IMMIP), University Hospital Bonn, Bonn, Germany

\section{Leander Sollberg}

Institute of Medical Microbiology, Immunology and Parasitology (IMMIP), University Hospital Bonn,

Bonn, Germany

\section{Michael Bawolt}

Institute of Medical Microbiology, Immunology and Parasitology (IMMIP), University Hospital Bonn, Bonn, Germany

\section{Christina Berens}

Institute of Medical Microbiology, Immunology and Parasitology (IMMIP), University Hospital Bonn, Bonn, Germany

\section{Peđa Kovačević}

Medical Intensive Care Unit, University Clinical Center of Republic of Srpska, Banja Luka, Bosnia and Herzegovina.

\section{Anika Trudić}

Faculty of Medicine, University of Novi Sad, Novi Sad, Serbia, Institute for Pulmonary Diseases of Vojvodina, Sremska Kamenica, Serbia

\section{Marijo Parcina}

Institute of Medical Microbiology, Immunology and Parasitology (IMMIP), University Hospital Bonn, Bonn, Germany

\section{Achim Hoerauf}

Institute of Medical Microbiology, Immunology and Parasitology (IMMIP), University Hospital Bonn, Bonn, Germany 
Keywords: Severe acute respiratory syndrome coronavirus 2 (SARS-CoV-2), specific antibody responses, neutralizing activity, Alpha immunoglobulin (IgA), Gamma immunoglobulin (IgG), Moderna, BioNTech, AstraZeneca, Sinopharm, Johnson and Johnson's, Sputnik-V, heterologous vaccination strategy.

Posted Date: December 29th, 2021

DOI: https://doi.org/10.21203/rs.3.rs-1197023/v1

License: (9) This work is licensed under a Creative Commons Attribution 4.0 International License. Read Full License 


\section{Abstract}

\section{BACKGROUND}

Severe acute respiratory syndrome coronavirus 2 (SARS-CoV-2) infection and the resulting coronavirus disease 2019 (COVID-19) have afflicted millions of people in a worldwide pandemic. Several vaccines have been developed to prevent infection and illness. However, the safety and efficacy of most of the vaccines currently available are still being questioned by part of the public opinion. Even if vaccineresistant individuals represent a minority in most countries, their hesitancy is sufficient to delay the highly desired 'herd-immunity threshold.'

\section{METHODS}

In an ongoing multinational trial, we collected blood samples from 365 adults, 18 years of age or older, vaccinated with mRNA vaccines (Moderna, BioNTech), viral DNA-vectored vaccines (AstraZeneca, Sputnik-V, and Johnson and Johnson), or the attenuated virus vaccine from Sinopharm.

Out of the 365 vaccinated individuals included in the study, 41 received two doses of Moderna Biotech's Spikevax, 92 received two doses of BioNTech's Comirnaty, 52 were vaccinated with two doses of OxfordAstraZeneca's Vaxzevria, 34 received one dose of Johnson and Johnson's Jansen, 35 two doses of Gamaleja's Sputnik-V and 28 two doses of Sinopharm's BBIBP-CorV. In addition, 40 received a prime dose of AstraZeneca followed by BioNTech as a booster, whereas 43 received Moderna's vaccine as a booster after a prime dose of AstraZeneca. After collecting reactogenicity data, the expression of S-Protein binding IgG and IgA were analyzed before and after full vaccination in each group using an automated sandwich ELISA system. In addition, the neutralizing capacity of sera from individuals from all groups was investigated using an ACE-2-RBD neutralizing assay.

\section{RESULTS}

The main side effects reported included short-term mild-to-moderate pain at the injection site, fatigue, and headache. More severe side effects were reported by vaccinees in the Moderna (10\%), AstraZeneca (11\%), Johnson and Johnson (5.9\%), and Sputnik-V (7.2\%) groups. No severe adverse reaction was reported in the BioNTech group, and the Sinopharm vaccinees presented the mildest reactogenicity profile, with $93.8 \%$ of the vaccinees declaring no adverse reactions.

Moderna's vaccine induced the highest amounts of SARS-CoV-2 specific $\lg G, \lg A$, and serum neutralization activity compared to the other groups. In contrast, people vaccinated with Sinopharm and Johnson and Johnson's vaccines have the lowest SARS-CoV-2-specific antibody titers. Vaccinees from the Johnson and Johnson group presented significant levels of SARS-CoV-2 specific IgA but not IgG compared to the controls before vaccination. In the Sinopharm group, neither IgG nor IgA expression was significant. In addition, sera from vaccinees of these two groups presented no significant neutralization potential compared to the unvaccinated controls. Significant negative correlations between age and SARS-CoV-2- specific IgG expression were observed in the Johnson and Johnson $(r=-0.4414, p=0.009)$ 
and Sinopharm ( $r=-0.6108, p=0.0006)$ groups. Remarkably, younger vaccinees (18-60 years old) in both Sinopharm and Johnson and Johnson groups produced substantial SARS-CoV-2 specific antibody expression and exhibited significant neutralization potential. While the AstraZeneca vaccine alone induced moderate IgG and IgA expression, the combination with Moderna or BioNTech mRNA vaccines induced higher antibody levels than a double dose of AstraZeneca and similar IgG expression and neutralization potential compared to Moderna, or BioNTech used alone.

\section{CONCLUSION}

The results suggest that the Moderna vaccine is the most immunogenic after two doses. AstraZeneca and Sputnik-V presented moderate but significant antibody expression and virus neutralizing properties. Low antibody and neutralization potential was observed in the elderly vaccinated with Sinopharm or Johnson and Johnson vaccines. The data also suggest that heterologous vaccination strategies combining the AstraZeneca DNA vectored vaccines and mRNA vaccines Moderna or BioNTech booster induced more robust antibody and virus neutralization potential compared to their homologous counterparts.

\section{Introduction}

Since it began in November 2019, the COVID-19 pandemic has caused significant morbidity and mortality worldwide and major social, educational, and economic disruptions. It is the most serious global health crisis ever experienced in modern history ${ }^{1}$. Seniors over 70 years and persons with comorbidities are at the highest risk for COVID-19 complications ${ }^{2}$. As of December 20 th, 2021, there have been 271963258 confirmed cases of infection, including 5331019 deaths $^{3}$. Recent data indicate increasing SARS-CoV-2 infection rates and COVID-19 in younger adults due to the occurrence of new virus variants ${ }^{4,5}$. In this context, vaccine rollout represents a tool of choice to fight the pandemic. Several groups developed vaccines to prevent the infection and control the pandemic. The amount of resources and the extraordinary speed of vaccine development against COVID-19 is unique in human history ${ }^{6}$. While the WHO declared COVID-19 a pandemic in March 2020, less than nine months later, more than 60 vaccines entered clinical trials, with 13 in Phase III clinical trials at the end of $2020^{7}$. New technologies, accumulated expertise during the development of vaccines against related viruses like MERS-CoV or SARS-CoV-1, as well as existing production platforms have made this fulminant acceleration possible ${ }^{8}$.

Currently available vaccines against COVID-19 include Messenger ribonucleic acid (mRNA) vaccines like Moderna's Spikevax and Pfizer-BioNTech's Comirnaty, recombinant adenovirus vectored vaccines such as Vaxzevria from Oxford-AstraZeneca, Janssen from Johnson \& Johnson Pharm, and Sputnik-V from the Gamaleya Research Center in Russia. The Chinese pharmaceutical firm Sinopharm opted for a more classical approach using inactivated viruses and proposed the BIBP COVID-19-vaccine, also known as BBIBP-CorV. All these vaccine candidates have been authorized for human use in many countries ${ }^{9}$. The approval of these vaccines offers a highly effective tool for the global control of the COVID-19 pandemic. While the high-speed vaccine development is undoubtedly a scientific and technological success, it has 
also raised concerns about safety and efficacy in the global population ${ }^{10}$. In this context, independent evaluations of the safety and effectiveness of these vaccines are highly needed.

Humoral immune responses and particularly neutralizing antibodies are key elements of the adaptive immunity against acutely cytopathic viruses such as the SARS-CoV-2 ${ }^{11-13}$. Gamma (IgG) and alpha (IgA) immunoglobulins are the first and second most abundant immunoglobins in human serum, respectively ${ }^{14}$. There is increasing evidence that neutralizing responses correlate with protection against COVID-19 ${ }^{15}$. Both IgG and IgA were reported to mediate viral neutralization in COVID-19 patients, and their neutralization potential is the key mechanism supporting the efficacy of convalescent plasma in the treatment of severe COVID-19 patients ${ }^{16-19}$. While $\lg \mathrm{G}$ is the main antibody in the blood and most tissues, IgA is the most abundant antibody at mucosal surfaces ${ }^{14,20}$, including the respiratory mucosa, main entry, and replication site of SARS-CoV-2 in the human body ${ }^{20-23}$. Investigations in influenza and SARS-CoV-2 infections suggested higher antiviral properties for $\lg A$ in comparison to $\lg \mathrm{G}^{24,25}$, suggesting a key role for IgA in protective immunity against SARS-CoV-2. However, limited data exist on IgG and IgA responses after COVID-19 vaccinations. In the present study, we compared SARS-CoV-2 spike antigenspecific serum IgA and IgG expression as well as virus neutralization potential in individuals vaccinated with five different COVID-19 vaccines, including mRNA vaccines from Pfizer-BioNTech and Moderna, viral DNA vectored vaccines from Johnson \& Johnson, Oxford-AstraZeneca, and Sputnik-V, as well as the attenuated virus vaccine from Sinopharm (BIBP COVID-19-vaccine).

\section{Material And Methods}

PARTICIPANTS, sample collection, and ethics

The study was conducted between January 2021 and October 2021 at the Institute of Medical Microbiology, Immunology and Parasitology (IMMIP) of the University Hospital of Bonn and is part of an ongoing survey. Volunteers were recruited in Bonn (Germany), in Sremska Kamenica (Serbia), and in Banja Luka (Bosnia and Herzegovina). A total of 365 (122 men and 243 women) were included in the study. Each volunteer recruited for the study gave informed consent to participate. Ethics approval for the study was obtained from the ethical board of the University Hospital Bonn (Lfd.Nr.439/20). Venous blood was collected 14-30 days after the last vaccination dose using the S-Monovette SERUM GEL blood collection system (Sarstedt AG, Nümbrecht, Germany). Donors with immune deficiency or using immunosuppressive treatment were excluded from the analyses. 


\begin{tabular}{|c|c|}
\hline \multicolumn{2}{|l|}{ COVID-19 vaccinated groups $(\mathrm{N}=365)$} \\
\hline \multicolumn{2}{|l|}{$2 \times$ Moderna $(\mathrm{N}=41)$} \\
\hline Mean age (Min-Max, Mean $\pm S D$ ) in years & $18-85(42.08 \pm 15.82)$ \\
\hline $\operatorname{Sex}(M / F)$ & $13 / 28$ \\
\hline \multicolumn{2}{|l|}{$2 \times$ BioNTech $(\mathrm{N}=92)$} \\
\hline Mean age (Min-Max, Mean $\pm S D$ ) in years & $20-88(44.32 \pm 18.51)$ \\
\hline $\operatorname{Sex}(M / F)$ & $37 / 55$ \\
\hline \multicolumn{2}{|l|}{ 2x AstraZeneca $(\mathrm{N}=52)$} \\
\hline Mean age (Min-Max, Mean $\pm S D$ ) in years & $20-82(42.90 . \pm 16.88)$ \\
\hline $\operatorname{Sex}(M / F)$ & $14 / 38$ \\
\hline \multicolumn{2}{|l|}{ 1x Johnson and Johnson (N=34) } \\
\hline Mean age (Min-Max, Mean $\pm S D$ ) in years & $20-71(35.48 \pm 15.65)$ \\
\hline $\operatorname{Sex}(M / F)$ & $23 / 11$ \\
\hline \multicolumn{2}{|l|}{ 2x Sputnik-V(N=35) } \\
\hline Mean age (Min-Max, Mean $\pm S D$ ) in years & $18-78(35.98 \pm 10.75)$ \\
\hline $\operatorname{Sex}(M / F)$ & $11 / 24$ \\
\hline \multicolumn{2}{|l|}{ 2x Sinopharm ( $\mathrm{N}=28)$} \\
\hline Mean age (Min-Max, Mean $\pm S D$ ) in years & $26-74(33.65 \pm 14.96)$ \\
\hline $\operatorname{Sex}(M / F)$ & $11 / 17$ \\
\hline \multicolumn{2}{|l|}{ 1x AstraZeneca + 1 x Moderna ( $\mathrm{N}=43)$} \\
\hline Mean age (Min-Max, Mean $\pm S D$ ) in years & $20-65(42.15 . \pm 20.24)$ \\
\hline $\operatorname{Sex}(M / F)$ & $6 / 37$ \\
\hline \multicolumn{2}{|l|}{1 x AstraZeneca +1 x BioNTech $(\mathrm{N}=40)$} \\
\hline Mean age (Min-Max, Mean $\pm S D$ ) in years & $20-98(45.92 . \pm 25.18)$ \\
\hline $\operatorname{Sex}(M / F)$ & $7 / 33$ \\
\hline \multicolumn{2}{|l|}{ Controls $(\mathrm{N}=30)$} \\
\hline \multicolumn{2}{|c|}{ Before vaccination $(\mathrm{N}=10)$, control unvaccinated $(\mathrm{N}=20)$} \\
\hline Mean age (Min-Max, Mean $\pm S D$ ) in years & $18-78(33.92 \pm 25.33)$ \\
\hline $\operatorname{Sex}(M / F)$ & $12 / 18$ \\
\hline
\end{tabular}




\section{COVID-19 vaccinated groups $(\mathrm{N}=365)$}

\section{Table.1 Epidemiological and clinical characteristics of study participants.}

REACTOGENICITY AND efficacy analyses

Reactogenicity investigations were done using questionnaires designed by the investigators and delivered to the participants. The survey was done onsite at the University Clinic of Bonn or the Zadar General Hospital of Zagreb, 2 to 3 weeks after each vaccine shot. Participants who agreed to participate gave written consent and filled a form with their demographics, including sex, age, earlier infection with SARSCoV-2, and the observed side effects. All responses were included anonymously.

Enzyme-Linked Immunosorbent Assay (ELISA) for the detection of SARS-COV-2 specific IgG and IgA

To detect the levels of SARS-CoV-2 specific antibodies, the Euroimmun SARS-CoV-2 IgG/IgA ELISA kit (Euroimmun, Lübeck, Germany) was used, according to the manufacturer's instructions. Serum samples were diluted 1:101 in the provided sample buffer and incubated at $37^{\circ} \mathrm{C}$ for $60 \mathrm{~min}$ in a 96 -well microtiter plate. Washings and incubation cycles were performed automatically using the predesigned program of Euroimmun's Analyzer I automate. Optical densities (OD) were measured at $450 \mathrm{~nm}$. SARS-CoV-2 specific immunoglobulin $\mathrm{G}$ and $\mathrm{A}$ expressions were calculated, and results were interpreted as per the manufacturer's protocol. Ratios $<0.8$ were considered as negative, $0.8-1.1$ as borderline and $>1.1$ as positive.

\section{Neutralization assay}

To test the neutralizing potential of SARS-CoV-2 specific antibodies in the sera of vaccinated individuals, SARS-CoV-2 antibody neutralizing immunoassay kits (ThermoFisher Scientific) were used according to the manufacturer's instructions. Briefly, $100 \mu \mathrm{L}$ of controls and 1:5 diluted sera from fully vaccinated or unvaccinated individuals were added to the wells of microplates pre-coated with SARS-CoV-2 receptorbinding domain (RBD) protein. The plates were then incubated for 1 hour to allow neutralizing antibodies present in the samples to bind to RBD specifically. After 3 washes with the provided wash solution, 100 $\mu \mathrm{L}$ of biotinylated $1 \mathrm{x}$ ACE2 and samples were incubated for an additional $1 \mathrm{~h}$. After incubation, samples were washed 3 times, and $100 \mu \mathrm{L}$ of $1 \times$ Streptavidin-HRP was added to each well. Plates were then incubated for an additional $30 \mathrm{~min}$, and $50 \mu \mathrm{L}$ of stop solution containing $2 \mathrm{~N} \mathrm{H} 2 \mathrm{SO} 4$ was added to stop the reaction. Signal development is indirectly proportional to the amount of specific neutralizing antibodies present. Plates were measured at $450 \mathrm{~nm}$ with the SpectraMax Pro plate reader (Molecular Devices), and neutralization potential was calculated according to the provided controls.

\section{Statistics}

Descriptive demographic and clinical data analyses are presented as mean \pm SD when continuous and as proportions (\%) when categorical. All graphs were generated using GraphPad Prism 8 (La Jolla, CA, USA). $\mathrm{p}$ values were calculated using the Kruskal Wallis test. Dunnett's multiple comparison test was used to 
compare all settings to the control (Unvaccinated), and Dunn's post hoc test was used to compare all groups. Significance is accepted if $p<0.05$.

\section{Results}

LOWest Systemic reactogenicity after vaccination with Sinopharm's BIBP COVID-19 vaccine

We first analyzed the reactogenicity of the different vaccines using an appropriate questionnaire. The most common local symptoms were pain at the site of injection and skin rash. Minor systemic side effects were seen in all groups. More severe systemic effects, including musculoskeletal symptoms, fever, and headache for more than 3 days, were observed in the Moderna (10\%), AstraZeneca (11\%), Johnson and Johnson (5.9\%), and Sputnik-V (7.2\%) groups. No severe systemic effect was reported in the BioNTech group. Sinopharm's BIBP COVID-19 vaccinees presented the lowest percentage of adverse reactions. Indeed, $93.2 \% \%$ of the participants who received this vaccine declared having experienced no systemic side effects (Figure 1).

Higher expression of SARS-CoV-2-specific IgG and IgA in mRNA vaccinated individuals

To determine whether SARS-CoV-2 specific antibody expression correlates with protection against the infection, we analyzed S-protein specific IgG and IgA in the sera of vaccinated individuals 3-6 weeks after full vaccination. The results indicate that mRNA vaccines generally induced the highest amounts of SARS-CoV-2-reactive IgG and IgA. The Moderna vaccine induced slightly more IgG and IgA compared to the BioNTech vaccine. AstraZeneca and Sputnik-V induced comparable amounts of SARS-CoV-2 specific IgG. However, IgA expression was higher in the Sputnik-V group compared to the AstraZeneca group. The IgG expression in these two groups was significantly higher compared to the unvaccinated controls but lower compared to both mRNA vaccines. In contrast, the amount of SARS-CoV-2-specific IgG in the Johnson and Johnson and Sinopharm groups was statistically comparable to the unvaccinated control group, $p>0.999$ and $p=0.860$, respectively. However, while SARS-CoV-2 specific IgA was significant in the Johnson \& Johnson group ( $p=0.004)$, no statistical difference was seen between Sinopharm and the control groups $(p=0.2287)$ in regard to SARS-CoV-2-specific IgA expression (Figure 2$)$.

low neutralizing potential in sera of Sinopharm and johnson and johnson vaccinated individuals

Next, we determined whether sera from vaccinated individuals can neutralize SARS-CoV-2 binding to the ACE-2 receptor. The data suggest Moderna and BioNTech groups exhibited the highest neutralization potential compared to the unvaccinated controls and all other groups. These data confirmed that mRNA COVID-19 vaccines induce antibodies with the highest neutralizing potential. In line with their antibody levels, AstraZeneca and Sputnik-V groups exhibited similar and significant neutralization potential compared to the unvaccinated controls. In contrast, no significant difference was seen between the Johnson and Johnson and Sinopharm groups and the control group in terms of SARS-CoV-2 neutralization potential (Figure 3). 
Significant negative correlation between age and IgG production in Johnson and Johnson and Sinopharm vaccinees

To define the impact of age on vaccine induced antibody production, Pearson's correlation test was used to investigate the relationship between the age of the vaccinees and the amplitude of SARS-CoV-2 specific IgA and IgG expression after full vaccination with Moderna, BioNTech, AstraZeneca, Johnson, and Johnson, Sputnik-V and Sinopharm vaccines. No significant correlation was seen between age and SARS-CoV-2 specific antibody expression in the Moderna group (Figure 4A-B). In the BioNTech group, a negative correlation was seen between age and IgG expression, and a trend was seen when correlating age and SARS-CoV-2 specific IgA expression (Figure 4C-D). Surprisingly, virus-specific IgA expression positively correlated with age in the AstraZeneca group, while no correlation with age was seen when considering IgG expression (Figure 4E-F). For Sputnik-V vaccinees, negative antibody-age correlation trends were observed for both IgA and IgG (Figure 4G-H). While no correlation was seen for IgA, a robust and significant negative correlation $(r=-0.4414, p=0.009)$ was seen between age and SARS-CoV-2specific IgG expression (Figure 4I-J). Similar trends were observed in the Sinopharm vaccinees' group where no significant correlation was seen between age and IgA. In contrast, a robust and significant negative correlation $(r=-0.6108, p=0.0006)$ was seen between age and virus-specific IgG expression (Figure 4K-L).

Low SARS-CoV-2 specific antibody Expression and neutralizing potential in older Johnson and Johnson and Sinopharm vaccinees

The strongest negative correlation between age and antibody expression was observed in the Johnson and Johnson and Sinopharm groups. To further analyze the impact of age on the humoral immune response in these groups, serum neutralizing activities were investigated in 3 different age groups: 18-40, 41-60, and 61-80+. The results indicated a significant reduction of neutralizing potential in older vaccinees. The lowest neutralization potential was observed in the 60-80+ years old groups (Figure 5).

Robust Antibody production and neutralization potential after AstraZeneca-mRNA vaccine combinations

We next compared the antibody responses with a double shot of AstraZeneca, Moderna, and BioNTech with the combinations AstraZeneca/Moderna or AstraZeneca/BioNTech. Our data showed that the combinations of AstraZeneca with a second dose of Moderna or BioNTech are significantly more effective at inducing SARS-CoV-2 specific IgG and IgA compared to 2 doses of AstraZeneca. In addition, IgA levels were higher in the homologous Moderna group compared to the AstraZeneca-Moderna group. No significant difference was seen between the AstraZeneca-BioNTech group and the homologous BioNTech group (Figure 6). These results were also reflected by the neutralization data. Indeed, neutralization potentials in sera of AstraZeneca/Modern and AstraZeneca/BioNTech groups were significantly higher compared to the groups who received 2 doses of AstraZeneca. In addition, while few individuals of the BioNTech group presented neutralization potentials lower than $70 \%$ in the AstraZeneca and BioNTech groups, all tested volunteers exhibited in the AstraZeneca/BioNTech group neutralizing potentials over $95 \%$ (Figure 7). 


\section{Discussion}

In this study based on 365 vaccinated participants, we showed that post-vaccine anti-spike IgG responses significantly vary according to the vaccine type. mRNA vaccines from Moderna and BioNTech induced the highest amount of spike-specific lgG and IgA antibodies and a high serum neutralization potential. High antibody levels in mRNA vaccinees were also observed in other studies ${ }^{26}$. The data suggest a high seroconversion and antibody-mediated virus neutralization potential in mRNA-vaccinated individuals.

Comparatively, DNA vectored vaccines Sputnik-V and AstraZeneca induced similar amounts of SARSCoV-2 specific IgG. Interestingly, higher IgA expression was seen in the Sputnik-V group compared to the AstraZeneca group. Both groups, however, presented similar neutralization potential. These data can be explained by the fact that Sputnik-V and the AstraZeneca vaccine are very similar in their conception and principle of action. For both vaccines, the gene coding for the SARS-CoV-2 spike protein is introduced into an adenovirus vector. The main difference between these two vector vaccines is that Sputnik-V uses two different vectors, the Ad26 for priming and Ad5 in the booster dose ${ }^{27}$, while the AstraZeneca includes the spike protein gene in the ChAdOx1 viral vector ${ }^{28,29}$.

Our results clearly showed a weaker performance for the Johnson and Johnson vaccine compared to mRNA and the other DNA vectored vaccines. Indeed, the Johnson and Johnson vaccine induced relatively low spike-specific IgG, and the sera of vaccinees from this group exhibit no significant neutralizing potential compared to the unvaccinated controls. Similar findings were obtained in a larger survey by Self et al., where postvaccination anti-spike and anti-RBD IgG levels were seen to be significantly lower in persons vaccinated with Johnson and Johnson than Moderna or Pfizer-BioNTech vaccines ${ }^{26}$. This weak performance can be explained by the fact that the Johnson and Johnson vaccine required only one dose. In line with our data, the American Food and Drug Administration (FDA) has recently amended the Emergency Use Authorizations (EUA) for the Janssen COVID-19 vaccine to include the use of a booster dose to be administrated to all recipients of the Johnson and Johnson vaccine. They may choose to receive either an additional full dose of Janssen's vaccine or a full dose of an mRNA vaccine ${ }^{30}$.

An even weaker performance was observed for the Sinopharm vaccine. In line with our data, a recent study in Bahrain showed that in a group of 22 persons vaccinated with a double dose of the Sinopharm vaccine, 20 were infected with SARS-CoV- $2^{31}$. Saeed et al., after analyzing the expression of spike-specific antibody levels in 2868 COVID-19 vaccinated individuals with the Sinopharm vaccine in Iran, came to the conclusion that two doses of Sinopharm may not be adequate to provide long-lasting immunity against SARS-CoV-2 ${ }^{32}$. It is, however, noticeable that the Sinopharm vaccine used whole inactivated SARS-CoV-2 viral particles ${ }^{33}$ so that our analyses of spike protein reactivity may miss the full extent of immune reactions to this vaccine. Further investigations are required to explore the responses to other viral proteins.

Nonetheless, correlation analyses of antigen-specific antibody expression with the age of the vaccinees revealed a strong negative correlation between age and antibody expression, especially in the Johnson 
and Johnson and Sinopharm groups. Further analyses revealed that younger vaccinees with 18-40 years in these groups exhibit significant antibody and neutralizing potential compared to older vaccinees. In the age group of 41-60-year-olds, the antibody expression and neutralizing activity were lower but still significant compared to the unvaccinated controls. Thus, while neutralization potential in 18-60 years old Johnson and Johnson and Sinopharm vaccinees was significant, no significance was seen when considering older adults (60-80+ years). These data suggest a higher risk of post-vaccination COVID-19 infection in this age category after Johnson and Johnson and Sinopharm vaccines. This finding, together with the emergence of new virus variants, is very worrying since this very same population of the elderly is considered at high risk of developing severe forms of COVID-19. The negative impact of immune senescence on vaccine efficacy is well known in both human and animal models ${ }^{34,35}$. In Influenza vaccination, for example, it was shown that age-dependent reduction of the expression of critical regulators of B cell maturation and class switch recombination such as Blimp-1, E47, and AID, leads to the production of fewer functional antibodies in the elderly ${ }^{36}$. Implications for the millions of people who received these two vaccines worldwide remain to be clarified. Booster doses with the more effective mRNA vaccines should be considered.

During our survey, the German Standing Vaccination Committee (STIKO), taking into account concerns after several reports of rare but serious blood clots in young adults ${ }^{37,38}$, recommended after AstraZeneca a second dose of one of the 2 available mRNA vaccines to individuals that received the first dose of AstraZeneca. We therefore also analyzed the antibody responses after this mixed vaccination strategy. The data clearly suggest a more robust SARS-CoV-2 specific IgG and IgA expression after this vaccination schedule. Similar data were found in Spain, where preliminary data on 600 AstraZeneca primed vaccinees demonstrated that a BioNTech second dose remarkably boosted antibody responses ${ }^{39}$. Our data further confirmed that the mix-and-match COVID-19 vaccination strategy triggered a stronger antibody production than two doses of a single vaccine. Noticeable was, however, that Moderna vaccinees conserved the highest SARS-CoV-2 specific IgA expression compared to all other groups.

The findings in this report are subject to three major limitations. First, antibody specificity and neutralization potential were not tested against emerging variants of concern. Indeed, an increasing concern is whether the vaccines currently available can protect against emerging SARS-CoV-2 variants 40-42. The study was largely performed before the predominance of the Delta (B.1.617.2) and the emergence of the Omicron variants in Europe ${ }^{5}$. Further investigations are required to analyze in-depth the antibody responses to different SARS-CoV-2 variants. Second, while our data focus on antibody responses, $T$ cell reactivity and $T$ cell memory might represent another important mechanism for longlasting vaccine-induced protection.

The present study compared the efficacy of the 6 major COVID-19 vaccines currently available (Moderna, BioNTech, AstraZeneca, Johnson and Johnson, Sputnik-V, and Sinopharm's COVID-19 vaccines). Our findings suggest that mRNA vaccines induced the highest titers of SARS-CoV-2 specific neutralizing antibodies. While all 6 vaccines have moderate reactogenicity and induce functional neutralizing 
antibodies in vaccinees, low antibody-mediated protection is seen in the elderly vaccinated with Sinopharm or Johnson and Johnson vaccines. Our data also demonstrated that heterologous vaccination strategies using priming with the AstraZeneca followed with a boost with an mRNA vaccine induced more robust antibody expression and virus neutralization potential compared to their homologous counterparts.

\section{Declarations}

\section{Declaration of interests}

The authors have declared that no competing interests exist.

\section{Role of funding source and ethics committee approval}

The funders had no role in study design, data collection, analysis, decision to publish, or manuscript preparation.

Ethical clearance for the study was given by the University Hospital Bonn's ethics committee.

\section{References}

1. Arabi YM, Murthy S, Webb S. COVID-19: a novel coronavirus and a novel challenge for critical care. Intensive Care Med 2020;46(5):833-836. DOI: 10.1007/s00134-020-05955-1.

2. Ramasamy MN, Minassian AM, Ewer KJ, et al. Safety and immunogenicity of ChAdOx1 nCoV-19 vaccine administered in a prime-boost regimen in young and old adults (COV002): a single-blind, randomised, controlled, phase 2/3 trial. Lancet 2021;396(10267):1979-1993. DOI: 10.1016/S01406736(20)32466-1.

3. WHO. Coronavirus Disease (COVID-19) Dashboard (2021). (https://covid19.who.int/).

4. Polack FP, Thomas SJ, Kitchin N, et al. Safety and Efficacy of the BNT162b2 mRNA Covid-19 Vaccine. N Engl J Med 2020;383(27):2603-2615. DOI: 10.1056/NEJMoa2034577.

5. Kandeel M, Mohamed MEM, Abd El-Lateef HM, Venugopala KN, El-Beltagi HS. Omicron variant genome evolution and phylogenetics. J Med Virol 2021. DOI: 10.1002/jmv.27515.

6. Ball P. The lightning-fast quest for COVID vaccines - and what it means for other diseases. Nature 2021;589(7840):16-18. DOI: 10.1038/d41586-020-03626-1.

7. WHO. The COVID-19 vaccine tracker and landscape compiles detailed information of each COVID-19 vaccine candidate in development by closely monitoring their progress through the pipeline. (https://www.who.int/publications/m/item/draft-landscape-of-covid-19-candidate-vaccines). 
8. Bok K, Sitar S, Graham BS, Mascola JR. Accelerated COVID-19 vaccine development: milestones, lessons, and prospects. Immunity 2021;54(8):1636-1651. DOI: 10.1016/j.immuni.2021.07.017.

9. He Q, Mao Q, Zhang J, et al. COVID-19 Vaccines: Current Understanding on Immunogenicity, Safety, and Further Considerations. Front Immunol 2021;12:669339. DOI: 10.3389/fimmu.2021.669339.

10. Wong LP, Alias $H$, Danaee $M$, et al. COVID-19 vaccination intention and vaccine characteristics influencing vaccination acceptance: a global survey of 17 countries. Infect Dis Poverty 2021;10(1):122. DOI: 10.1186/s40249-021-00900-w.

11. Dorner T, Radbruch A. Antibodies and B cell memory in viral immunity. Immunity 2007;27(3):384-92. DOI: 10.1016/j.immuni.2007.09.002.

12. Ellinger B, Bojkova D, Zaliani A, et al. A SARS-CoV-2 cytopathicity dataset generated by high-content screening of a large drug repurposing collection. Sci Data 2021;8(1):70. DOI: 10.1038/s41597-02100848-4.

13. Zhu N, Wang W, Liu Z, et al. Morphogenesis and cytopathic effect of SARS-CoV-2 infection in human airway epithelial cells. Nat Commun 2020;11(1):3910. DOI: 10.1038/s41467-020-17796-z.

14. Vidarsson G, Dekkers G, Rispens T. IgG subclasses and allotypes: from structure to effector functions. Front Immunol 2014;5:520. DOI: 10.3389/fimmu.2014.00520.

15. Khoury DS, Cromer D, Reynaldi A, et al. Neutralizing antibody levels are highly predictive of immune protection from symptomatic SARS-CoV-2 infection. Nat Med 2021;27(7):1205-1211. DOI: 10.1038/s41591-021-01377-8.

16. Wang Z, Lorenzi JCC, Muecksch F, et al. Enhanced SARS-CoV-2 neutralization by dimeric IgA. Sci Transl Med 2021;13(577). DOI: 10.1126/scitransImed.abf1555.

17. Klingler J, Weiss S, Itri V, et al. Role of IgM and IgA Antibodies in the Neutralization of SARS-CoV-2. medRxiv 2020. DOI: 10.1101/2020.08.18.20177303.

18. Maeda K, Higashi-Kuwata N, Kinoshita N, et al. Neutralization of SARS-CoV-2 with IgG from COVID19-convalescent plasma. Sci Rep 2021;11(1):5563. DOI: 10.1038/s41598-021-84733-5.

19. Salazar E, Kuchipudi SV, Christensen PA, et al. Convalescent plasma anti-SARS-CoV-2 spike protein ectodomain and receptor-binding domain IgG correlate with virus neutralization. J Clin Invest 2020;130(12):6728-6738. DOI: 10.1172/JCl141206.

20. Terauchi Y, Sano K, Ainai A, et al. IgA polymerization contributes to efficient virus neutralization on human upper respiratory mucosa after intranasal inactivated influenza vaccine administration. Hum Vaccin Immunother 2018;14(6):1351-1361. DOI: 10.1080/21645515.2018.1438791.

21. Ding Q, Lu P, Fan Y, Xia Y, Liu M. The clinical characteristics of pneumonia patients coinfected with 2019 novel coronavirus and influenza virus in Wuhan, China. J Med Virol 2020;92(9):1549-1555. DOI: 10.1002/jmv.25781.

22. Pan $\mathrm{Y}$, Guan $\mathrm{H}$, Zhou S, et al. Initial CT findings and temporal changes in patients with the novel coronavirus pneumonia (2019-nCoV): a study of 63 patients in Wuhan, China. Eur Radiol 2020;30(6):3306-3309. DOI: 10.1007/s00330-020-06731-x. 
23. Zhu N, Zhang D, Wang W, et al. A Novel Coronavirus from Patients with Pneumonia in China, 2019. N Engl J Med 2020;382(8):727-733. DOI: 10.1056/NEJMoa2001017.

24. Muramatsu M, Yoshida R, Yokoyama A, et al. Comparison of antiviral activity between IgA and IgG specific to influenza virus hemagglutinin: increased potential of $\lg A$ for heterosubtypic immunity. PLoS One 2014;9(1):e85582. DOI: 10.1371/journal.pone.0085582.

25. Sterlin D, Mathian A, Miyara M, et al. IgA dominates the early neutralizing antibody response to SARS-CoV-2. Sci TransI Med 2021;13(577). DOI: 10.1126/scitranslmed.abd2223.

26. Self WH, Tenforde MW, Rhoads JP, et al. Comparative Effectiveness of Moderna, Pfizer-BioNTech, and Janssen (Johnson \& Johnson) Vaccines in Preventing COVID-19 Hospitalizations Among Adults Without Immunocompromising Conditions - United States, March-August 2021. MMWR Morb Mortal Wkly Rep 2021;70(38):1337-1343. DOI: 10.15585/mmwr.mm7038e1.

27. Cazzola M, Rogliani P, Mazzeo F, Matera MG. Controversy surrounding the Sputnik V vaccine. Respir Med 2021;187:106569. DOI: 10.1016/j.rmed.2021.106569.

28. Barrett JR, Belij-Rammerstorfer S, Dold C, et al. Author Correction: Phase 1/2 trial of SARS-CoV-2 vaccine ChAdOx1 nCoV-19 with a booster dose induces multifunctional antibody responses. Nat Med 2021;27(6):1113. DOI: 10.1038/s41591-021-01372-z.

29. Barrett JR, Belij-Rammerstorfer S, Dold C, et al. Phase $1 / 2$ trial of SARS-CoV-2 vaccine ChAdOx1 nCoV-19 with a booster dose induces multifunctional antibody responses. Nat Med 2021;27(2):279288. DOI: 10.1038/s41591-020-01179-4.

30. FDA. Coronavirus (COVID-19) Update: FDA Takes Additional Actions on the Use of a Booster Dose for COVID-19 Vaccines. (https://www.fda.gov/news-events/press-announcements/coronavirus-covid-19update-fda-takes-additional-actions-use-booster-dose-covid-19-vaccines).

31. Jahromi M, Al Sheikh MH. Partial protection of Sinopharm vaccine against SARS COV2 during recent outbreak in Bahrain. Microb Pathog 2021;158:105086. DOI: 10.1016/j.micpath.2021.105086.

32. Saeed U, Uppal SR, Piracha ZZ, Uppal R. SARS-CoV-2 Spike Antibody Levels Trend among Sinopharm Vaccinated People. Iran J Public Health 2021;50(7):1486-1487. DOI: 10.18502/ijph.v50i7.6640.

33. Wang H, Zhang $\mathrm{Y}$, Huang B, et al. Development of an Inactivated Vaccine Candidate, BBIBP-CorV, with Potent Protection against SARS-CoV-2. Cell 2020;182(3):713-721 e9. DOI:

10.1016/j.cell.2020.06.008.

34. Lang PO, Govind S, Bokum AT, et al. Immune senescence and vaccination in the elderly. Curr Top Med Chem 2013;13(20):2541-50. DOI: 10.2174/15680266113136660181.

35. Coe CL, Lubach GR, Kinnard J. Immune senescence in old and very old rhesus monkeys: reduced antibody response to influenza vaccination. Age (Dordr) 2012;34(5):1169-77. DOI: 10.1007/s11357011-9356-8.

36. Frasca D, Diaz A, Romero M, Blomberg BB. The generation of memory B cells is maintained, but the antibody response is not, in the elderly after repeated influenza immunizations. Vaccine 2016;34(25):2834-40. DOI: 10.1016/j.vaccine.2016.04.023. 
37. Graham F. Daily briefing: European regulator links AstraZeneca vaccine to rare blood clots. Nature 2021. DOI: 10.1038/d41586-021-00932-0.

38. Wise J. Covid-19: European countries suspend use of Oxford-AstraZeneca vaccine after reports of blood clots. BMJ 2021;372:n699. DOI: 10.1136/bmj.n699.

39. Callaway E. Mix-and-match COVID vaccines trigger potent immune response. Nature 2021;593(7860):491. DOI: 10.1038/d41586-021-01359-3.

40. Supasa P, Zhou D, Dejnirattisai W, et al. Reduced neutralization of SARS-CoV-2 B.1.1.7 variant by convalescent and vaccine sera. Cell 2021;184(8):2201-2211 e7. DOI: 10.1016/j.cell.2021.02.033.

41. Ho D, Wang P, Liu L, et al. Increased Resistance of SARS-CoV-2 Variants B.1.351 and B.1.1.7 to Antibody Neutralization. Res Sq 2021. DOI: 10.21203/rs.3.rs-155394/v1.

42. Wang P, Nair MS, Liu L, et al. Antibody resistance of SARS-CoV-2 variants B.1.351 and B.1.1.7. Nature 2021;593(7857):130-135. DOI: 10.1038/s41586-021-03398-2.

\section{Figures}

A

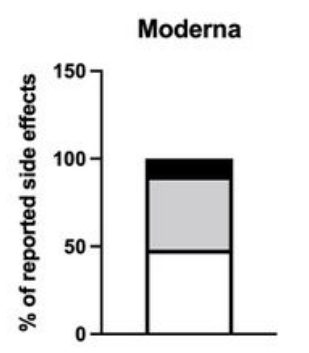

D Johnson and Johnson

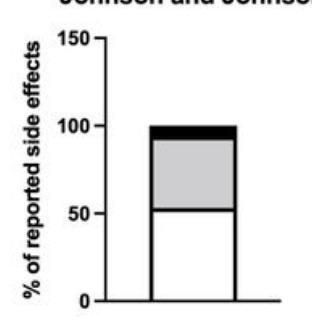

B

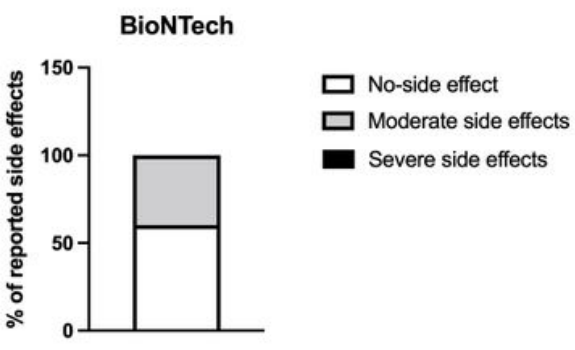

E

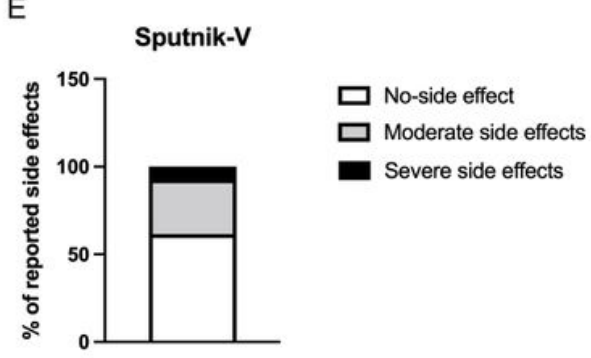

C

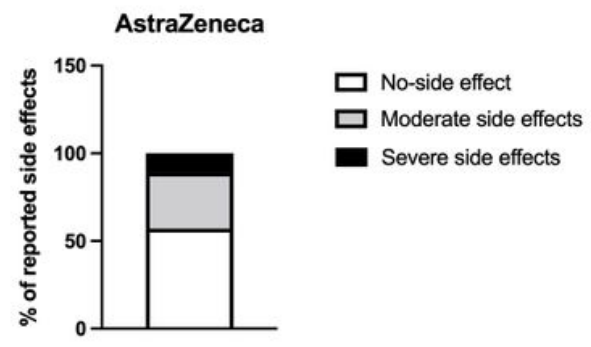

$\mathrm{F}$

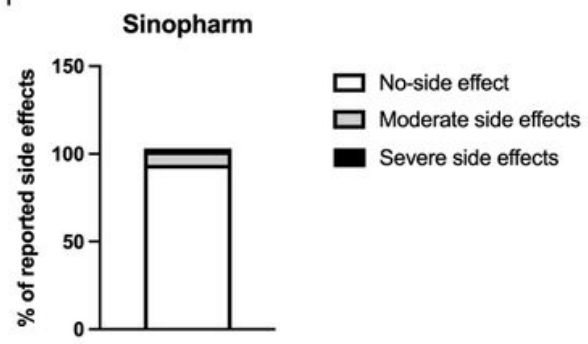

\section{Figure 1}

Level of reported systemic adverse reactions. Graphs represent the proportion of individuals presenting side effects in each group. The white parts of the histograms indicate the percentage of persons with no systemic side effects. The grey portions represent the percentage of people with mild side effects, while the dark parts indicate the percentages of individuals with severe side effects. 
A

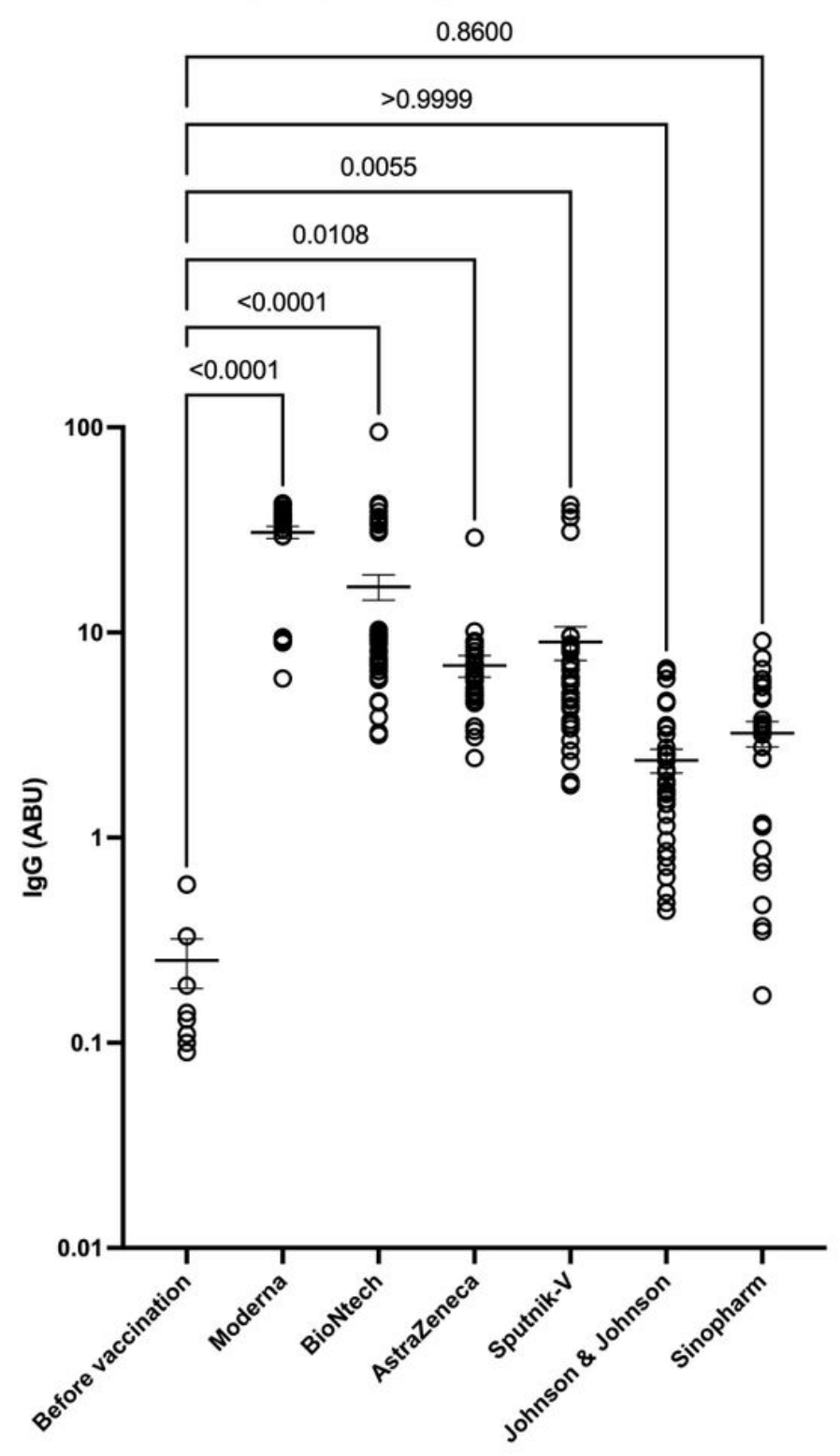

B

Antigen specific $\lg A$ after full vaccination

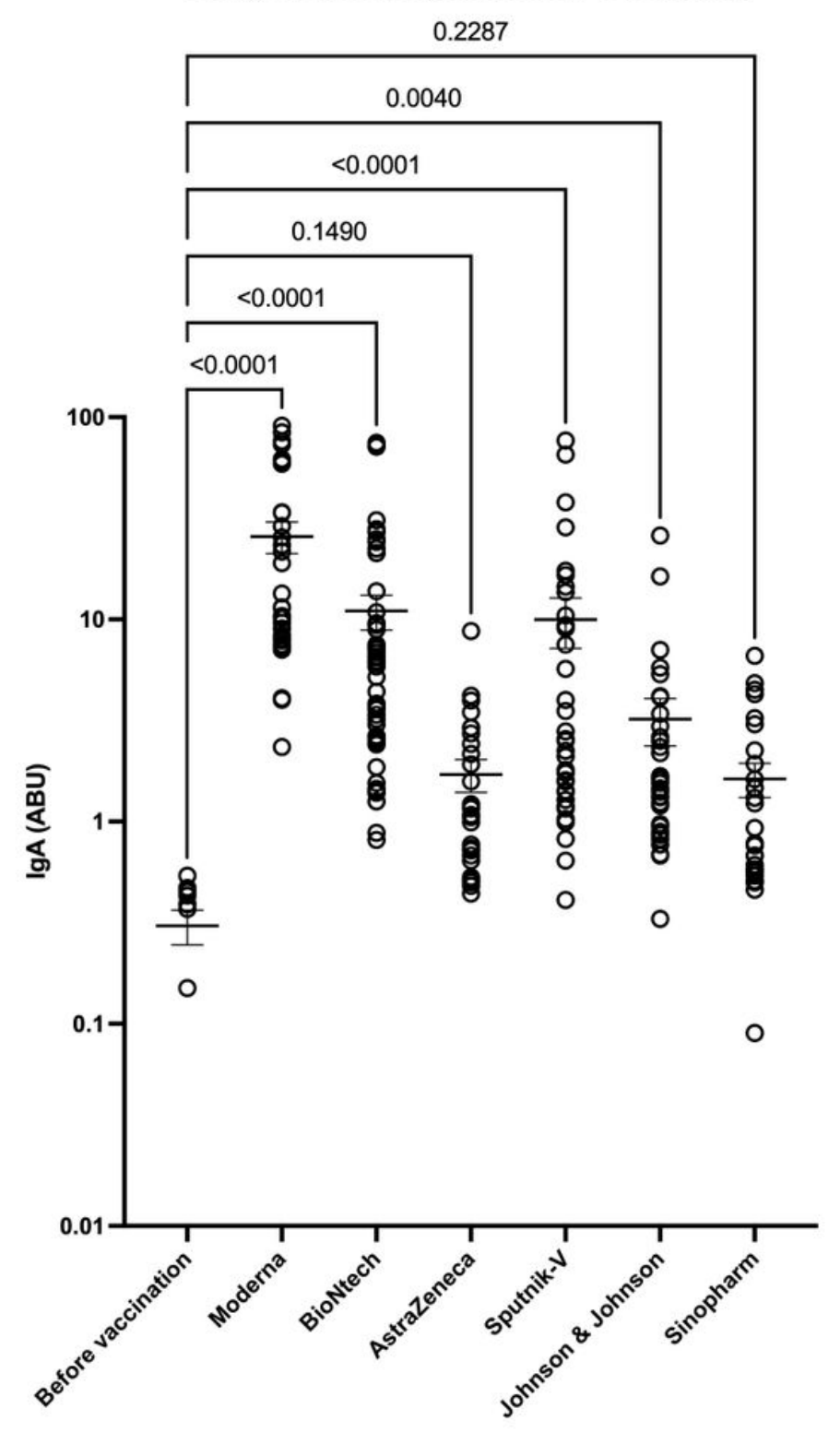

Figure 2

Higher expression of SARS-CoV-2 reactive IgG and IgA after immunization mRNA vaccines. Graphs represent the expression SARS-CoV-2 spike-protein binding $\lg G(A)$ and $\lg A(B)$ in arbitrary binding units $(A B U)$. Each symbol represents individual donors. Indicated $p$ values were calculated using the KruskalWallis test followed by Dunnett's post hoc to compare each group to the controls before vaccination. Bars represent mean \pm SEM. Significance is accepted if $p<0.05$. 


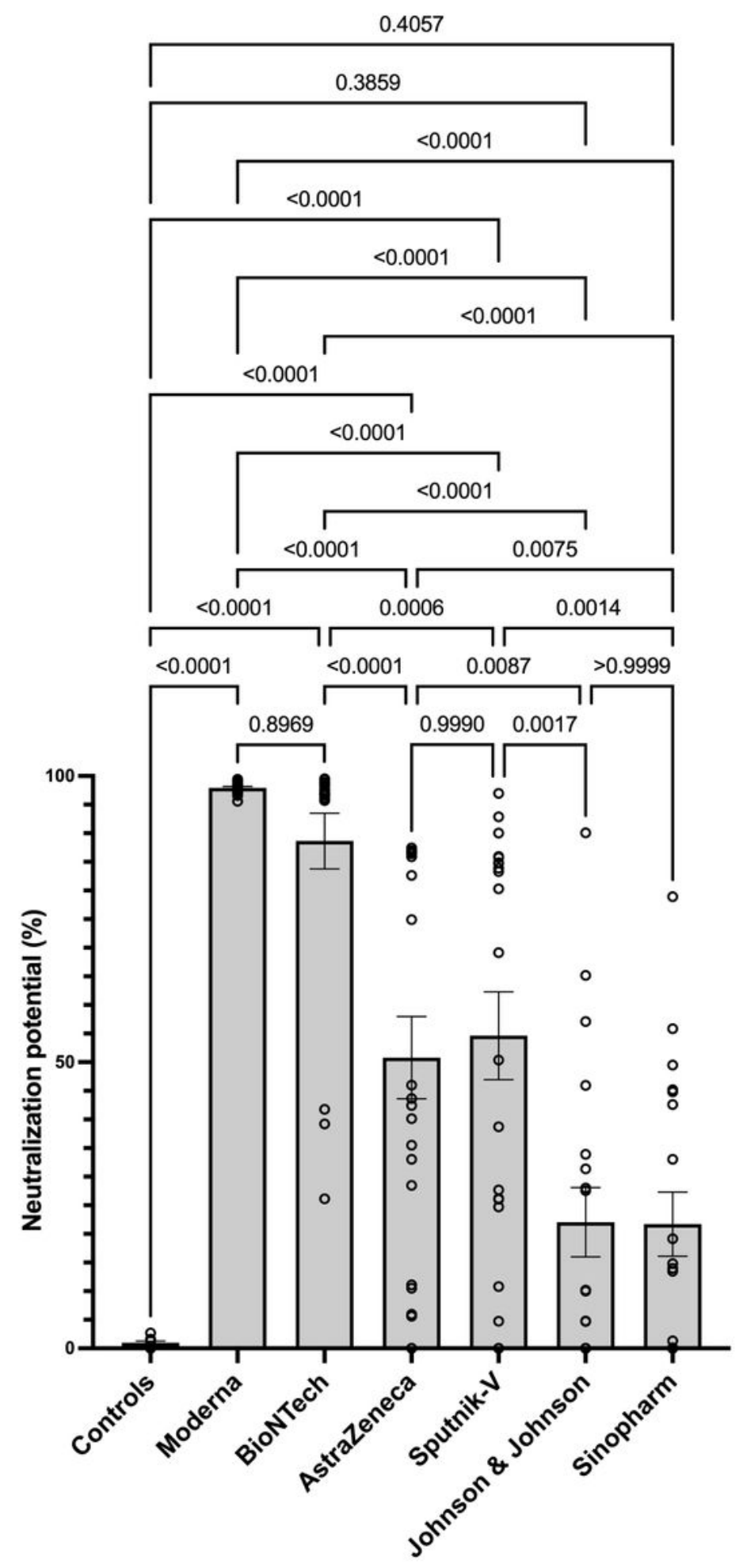

Figure 3

Higher neutralizing potential of mRNA vaccines in mRNA-vaccinated individuals. The graph represents the percentage of neutralization observed using sera from each vaccination group. Each symbol represents individual donors. Indicated $p$ values were calculated using Kruskal-Wallis 'test followed by Dunn's comparison post hoc to compare all groups. Bars represent the mean \pm SEM percentage of neutralization. Significance is accepted if $p<0.05$. 


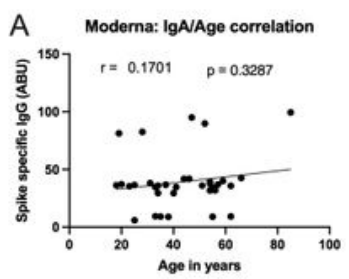

C

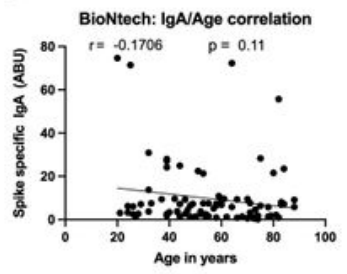

E

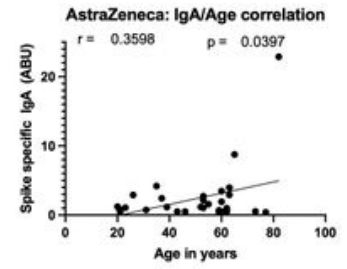

G

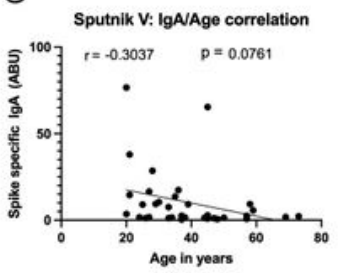

I

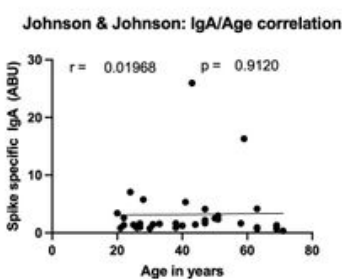

K

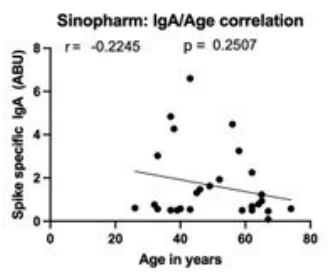

B Moderna: Ig $\mathrm{G} / \mathrm{Age}$ correlation

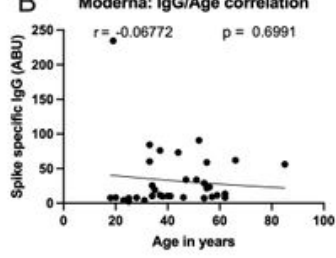

D

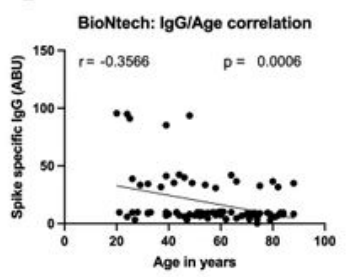

F

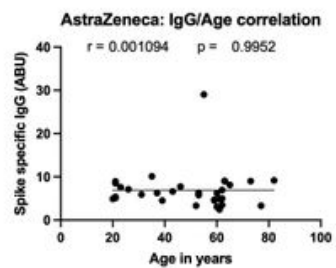

H Sputnik V: IgG/Age correlation

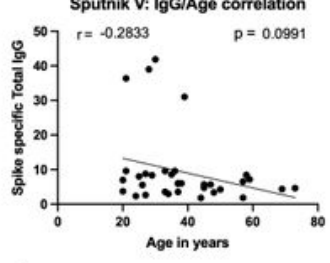

J

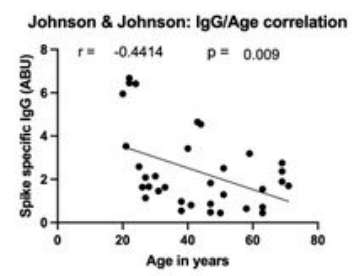

L

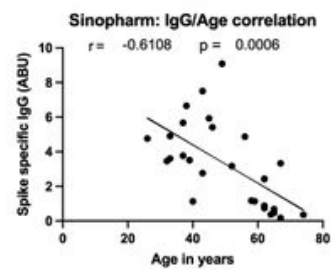

Figure 4

Robust age-dependent decrease of vaccine-induced antibody production in Johnson and Sinopharm vaccinees. Graphs represent the correlation of SARS-CoV-2 specific spike-protein binding IgG and IgA with age. Dots represent individual donors. Indicated $r$ and $p$ values were calculated using the Pearson $r$ correlation analysis. Significance is accepted if $p<0.05$. 

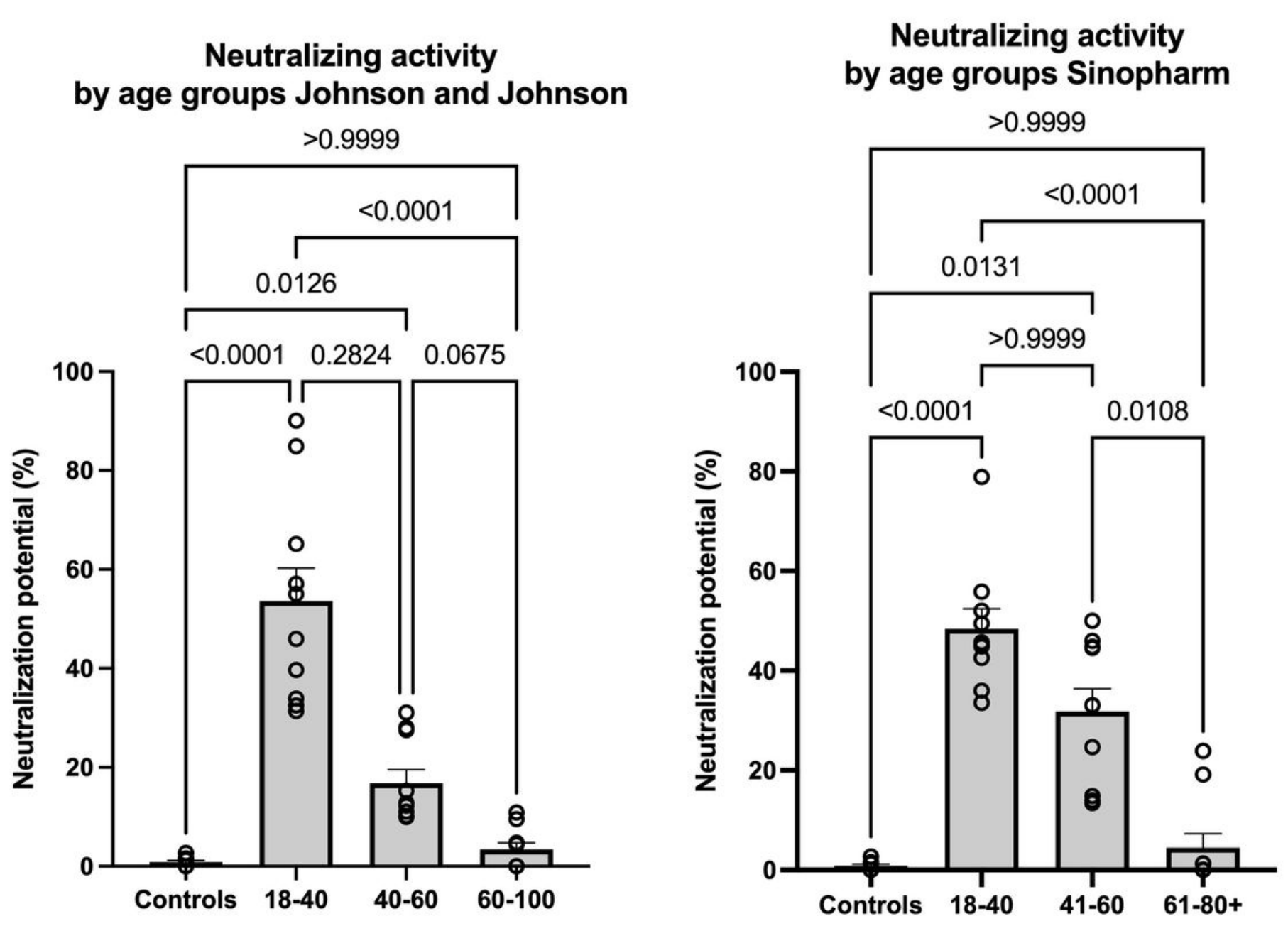

Figure 5

Low neutralization potential in elderly vaccinees. Graphs represent percentages of neutralization observed using sera from Johnson and Johnson (A) and Sinopharm (B) vaccinees groups in 3 different age categories: 18-40, 41-60, and 61-80+ years old. Each symbol represents individual donors. Indicated $p$ values were calculated using Kruskal-Wallis 'test followed by Dunn's comparison post hoc to compare all groups. Bars represent the mean percentages of neutralization \pm SEM. Significance is accepted if $p<0.05$. 

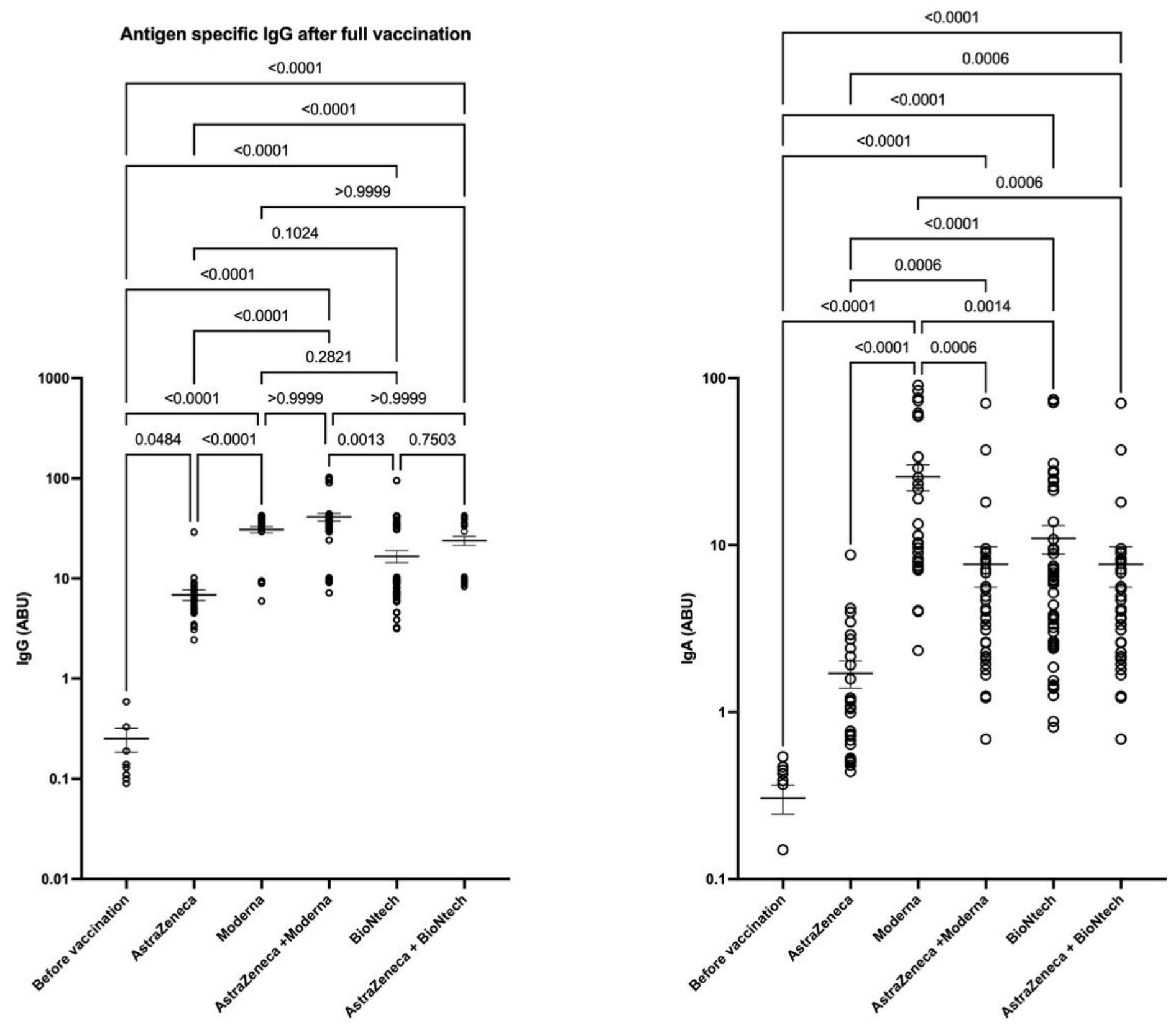

Figure 6

High antibody responses in AstraZeneca- mRNA vaccine combinations. Graphs represent the expression SARS-CoV-2 specific spike-protein binding $\operatorname{IgG}(A)$ and $\lg A(B)$ in people that received homologous vaccine doses (AstraZeneca, Moderna, or BioNTech) 's COVID-19 vaccines or the heterologous combinations (AstraZeneca-Moderna or AstraZeneca-BioNTech). Each symbol represents individual donors. Indicated p values were calculated using Kruskal-Wallis 'test followed by Dunn's comparison post hoc to compare all groups. Bars represent the means \pm SEM SARS-CoV-2 spike protein-specific antibody expression in arbitrary binding units (ABU). Significance is accepted if $p<0.05$. 
Neutralizing Potential all vaccines

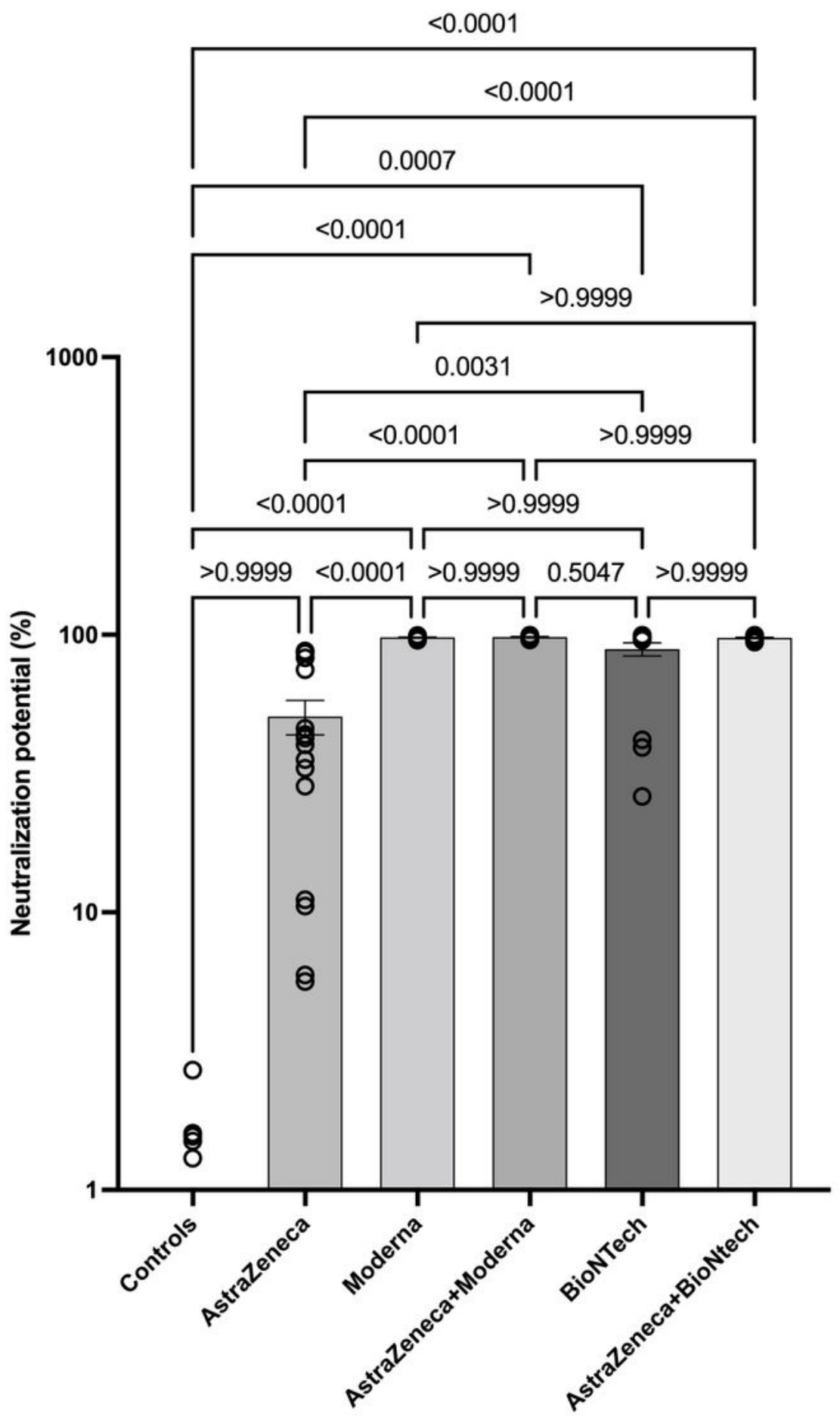

Figure 7

Higher neutralization potential in AstraZeneca-mRNA vaccine combinations. The graph represents the percentage of neutralization observed in the sera from groups of unvaccinated individuals (Controls), people that received homologous vaccine doses (AstraZeneca, Moderna, or BioNTech) 's COVID-19 vaccines or the heterologous combinations (AstraZeneca-Moderna or AstraZeneca-BioNTech). Each symbol represents individual donors. Indicated p values were calculated using Kruskal- 
Wallis 'test followed by Dunn's comparison post hoc to compare all groups. Bars represent the means \pm SEM percentage of neutralization. Significance is accepted if $p<0.05$. 